Cahiers
de Recherche $_{\text {sur les }}$ Droits

Cahiers de la recherche sur les droits

Fondamentaux fondamentaux

16 | 2018

Les partis politiques

\title{
Chronique de jurisprudence de la Cour européenne des droits de l'homme relative aux prisons 2016-2017
}

A Chronicle of the Case Law of the European Court of Human Rights Concerning Prisons 2016-2017

Jean-Manuel Larralde

\section{OpenEdition \\ Journals}

Édition électronique

URL : https://journals.openedition.org/crdf/339

DOI : $10.4000 /$ crdf.339

ISSN : 2264-1246

Éditeur

Presses universitaires de Caen

Édition imprimée

Date de publication : 16 novembre 2018

Pagination : 187-196

ISBN : 978-2-84133-901-3

ISSN : $1634-8842$

Référence électronique

Jean-Manuel Larralde, «Chronique de jurisprudence de la Cour européenne des droits de l'homme relative aux prisons 2016-2017 ", Cahiers de la recherche sur les droits fondamentaux [En ligne], 16 | 2018, mis en ligne le 16 novembre 2019, consulté le 14 novembre 2022. URL : http://

journals.openedition.org/crdf/339; DOI : https://doi.org/10.4000/crdf.339 


\title{
Chronique de jurisprudence de la Cour européenne des droits de l'homme relative aux prisons 2016-2017
}

\author{
Jean-Manuel LARRALDE \\ Professeur de droit public à l'université de Caen Normandie \\ Centre de recherche sur les droits fondamentaux et les évolutions du droit (CRDFED, EA 2132)
}

\begin{abstract}
I. La lutte contre les mauvaises conditions de détention
II. La protection de la santé et de la qualité des soins

III. Le contrôle des violences en détention

IV. Le respect de la vie privée et familiale et des relations avec l'extérieur

V. L'existence de voies de recours effectives
\end{abstract}

Si la Cour européenne des droits de l'homme n'a pas rendu en 2014-2015 de jurisprudence spectaculaire ou totalement inattendue concernant les prisons et les droits des personnes privées de leur liberté, elle a néanmoins continué à approfondir les thématiques posées par l'arrêt Kudla c. Pologne du 26 octobre 2000, qui impose aux États «de s'assurer que tout prisonnier est détenu dans des conditions qui sont compatibles avec le respect de la dignité humaine », ce qui implique que « eu égard aux exigences pratiques de l'emprisonnement, la santé et le bien-être du prisonnier [doivent être] assurés de manière adéquate $»^{1}$. De nouveaux arrêts renvoient à des contentieux conventionnels variés, qui relèvent essentiellement de la prohibition de la torture et des peines ou traitements inhumains ou dégradants (article 3 de la Convention européenne des droits de l'homme), mais concernent aussi le droit à la vie (article 2), la protection du droit à la vie privée et familiale (article 8), le droit au recours (article 13), ou encore le droit à la sûreté (article 5)...

L'abondante jurisprudence strasbourgeoise a permis d'apporter d'importantes précisions sur les méthodes retenues par la Cour dans le cadre de la lutte contre les mauvaises conditions de détention (I). Mais on retrouve aussi les fortes exigences strasbourgeoises en matière de protection de la santé des détenus et de la qualité des soins en détention (II), ainsi qu'une volonté ferme de lutter contre les violences en prison (III). Outil indispensable à la lutte contre la désocialisation des personnes privées de leur liberté, le respect de la vie privée et familiale ainsi que la garantie des relations avec l'extérieur font également l'objet d'une vigilance particulière des juges strasbourgeois (IV). Ces derniers n'oublient pas davantage que les détenus sont aussi des requérants, qui doivent pouvoir accéder à des recours effectifs afin de protéger leurs droits (V).

\section{La lutte contre les mauvaises conditions de détention}

Le contrôle de la dignité des conditions de détention constitue un axe fort de la jurisprudence strasbourgeoise 
depuis le début de la décennie 2000. À cet égard, 2016 a permis la clarification des méthodes employées par la Cour dans les situations de détention indignes, notamment générées par le surpeuplement carcéral, grâce aux salutaires précisions apportées par la grande chambre dans son arrêt du 20 octobre 2016, Muršić c. Croatie. Tout en refusant de donner

[...] une fois pour toutes la mesure chiffrée de l'espace personnel qui doit être octroyé à un détenu pour que ses conditions de détention puissent être jugées compatibles avec la Convention au regard de l'article $3^{2}$.

\section{La Cour confirme toutefois}

[...] que l'exigence de $3 \mathrm{~m}^{2}$ de surface au sol par détenu en cellule collective doit demeurer la norme minimale pertinente aux fins de l'appréciation des conditions de détention au regard de l'article 3 de la Convention ${ }^{3}$.

En conséquence, lorsque la surface au sol disponible s'avère inférieure à $3 \mathrm{~m}^{2}$, ce manque d'espace personnel est considéré comme étant à ce point grave qu'il donne lieu à une forte présomption de violation de l'article 3 . La grande chambre précise toutefois que, dans de tels cas, il est possible que la violation de l'article 3 de la Convention ne soit pas reconnue, car il existe des «facteurs susceptibles de compenser le manque d'espace personnel ${ }^{4}$. Le renversement de la présomption (qui n'est toutefois possible que pour des situations «courtes, occasionnelles et mineures ${ }^{5}$ ) est effectué par les juges européens si la détention s'est accompagnée d'une liberté de circulation suffisante dans les parties communes et d'activités hors cellule adéquates et si le détenu est incarcéré dans un établissement offrant des conditions de détention décentes ${ }^{6}$. Par ailleurs, lorsqu'un détenu dispose dans la cellule d'un espace personnel compris entre 3 et $4 \mathrm{~m}^{2}$, la violation de l'article 3 pourra être retenue

[...] si le manque d'espace s'accompagne d'autres mauvaises conditions matérielles de détention, notamment d'un défaut d'accès à la cour de promenade ou à l'air et à la lumière naturels, d'une mauvaise aération, d'une température insuffisante ou trop élevée dans les locaux, d'une absence d'intimité aux toilettes ou de mauvaises conditions sanitaires et hygiéniques?

Enfin, lorsque le détenu dispose de plus de $4 \mathrm{~m}^{2}$ d'espace personnel, il ne s'agit plus aux yeux de la Cour d'une situation de surpopulation carcérale. La violation de l'article 3 pourra toutefois être toujours retenue si les conditions matérielles de détention sont dégradées ${ }^{8}$.

La Cour de Strasbourg a eu depuis lors l'occasion de faire régulièrement application de cette jurisprudence de principe. Ainsi, dans l'arrêt Sklyar c. Russie du 18 juillet 2017, elle conclut à une violation de l'article 3 de la Convention, en relevant des conditions sanitaires inadéquates en cellule, mais surtout en condamnant le manque d'espace personnel disponible pour chaque détenu (inférieur à $2 \mathrm{~m}^{2}$ ), insuffisant et entraînant un manque d'intimité, enduré qui plus est pendant une période prolongée ${ }^{9}$. De même, dans l'arrêt Sylla et Nollomont c. Belgique du 16 mai 2017, la Cour relève que le premier requérant disposait certes d'un espace théorique au sol de $3 \mathrm{~m}^{2}$, mais dans le calcul duquel il convenait d'inclure l'espace occupé par les meubles pour déterminer l'espace personnel effectif ${ }^{\circ}$. Le requérant possédait donc en pratique un espace personnel inférieur à $3 \mathrm{~m}^{2}$, constituant donc une forte présomption de violation de l'article 3, violation confirmée par les mauvaises conditions de détention (en l'espèce, un accès à la cour de promenade limité à une heure par jour, aucune autre activité hors cellule n'étant prévue, une possibilité de se doucher restreinte à deux fois par semaine, un accès à la seule eau froide en raison de pannes, des vêtements, draps et serviettes changés seulement toutes les trois semaines). Quant à M. Nollomont, si celui-ci a pu bénéficier d'un espace cellulaire personnel de $4,4 \mathrm{~m}^{2}$, sa détention a également constitué un traitement inhumain et dégradant,

[...] du fait de la combinaison d'un régime pauvre en activités extérieures à la cellule, et au sein de la cellule, de l'exposition au tabagisme passif ainsi que du manque d'intimité dans l'usage des toilettes ${ }^{11}$.

À l'inverse, dans son arrêt Radzhab Magomedov c. Russie du 15 décembre 2016, les juges passent en revue les conditions matérielles de détention: les conditions

2. Cour EDH, 20 octobre 2016, Muršić c. Croatie, $\mathrm{n}^{\circ}$ 7334/13, $\$ 103$.

3. Ibid., $\$ 110$.

4. Ibid., $\$ 119$ sq.

5. Ibid., $\$ 130$ et 138 .

6. Ibid.

7. Ibid., $\$ 139$; voir aussi $\$ 106$. La liste n’étant pas limitative, puisque la Cour vérifie également l'équipement des cours de promenade (offrant la possibilité réelle de se dépenser physiquement et aménagées de façon à permettre le repos et pourvues d'un abri protégeant contre les intempéries), le degré de vétusté des locaux, la présence de nuisibles et/ou de parasites, la cohabitation avec des codétenus atteints de maladies contagieuses, la qualité et la quantité de la nourriture...

8. L'arrêt Muršić ne lève toutefois pas l'ensemble des difficultés. Comme le rappellent en effet les juges Sajó, López Guerra et Wojtyczek dans leur opinion partiellement dissidente $(\$ 5)$, plusieurs organes internationaux ont traité différemment la question de l'espace personnel en prison: le Comité international de la Croix-Rouge recommande ainsi un minimum de 5,4 $\mathrm{m}^{2}$ par détenu en cellule individuelle et 3,4 $\mathrm{m}^{2}$ en cellule collective. Le Comité européen pour la prévention de la torture a quant à lui retenu $6 \mathrm{~m}^{2}$ d'espace personnel en cellule individuelle et $4 \mathrm{~m}^{2}$ par détenu en cellule collective. Ceci aboutit à la reconnaissance d'un double standard du Conseil de l'Europe, qui est de nature à fragiliser la portée des travaux des différents organes strasbourgeois en la matière (voir sur ce point l'opinion partiellement dissidente du juge Pinto de Albuquerque).

9. Cour EDH, 18 juillet 2017, Sklyar c. Russie, $\mathrm{n}^{\circ}$ 45498/11. Pour un raisonnement semblable, voir Cour EDH, 21 juin 2016, G. c. Russie, $\mathrm{n}^{\circ}$ 42526/o7; 5 octobre 2017, Ābele c. Lettonie, $\mathrm{n}^{\circ}$ 60429/12 et 72760/12.

10. Cour EDH, 16 mai 2017, Sylla et Nollomont c. Belgique, $\mathrm{n}^{\circ} 37768 / 13$ et 36467/14, $\$ 26$ sq.

11. Ibid., $\$ 41$. Pour un raisonnement semblable, voir, inter alia, Cour EDH, 3 octobre 2017, Alexandru Enache c. Roumanie, ${ }^{\circ} 16986 / 12$. 
sanitaires et d'hygiène sont jugées certes non irréprochables, mais appropriées (un mur de brique séparant les toilettes du reste de la cellule, de l'eau froide disponible, un accès quotidien aux douches, des draps changés chaque semaine...). Le requérant pouvait en outre accéder à une heure de promenade quotidienne. Même si le gouvernement n'a pas contesté la présence de punaises de lit et des températures froides dans les cellules $^{12}$, la Cour considère que de telles conditions de détention, certes loin d'être idéales, n'atteignent pas le degré de sévérité nécessaire pour caractériser une violation de l'article 3. De même, la possibilité de passer un très long temps hors de la cellule permet à l'État d'éviter une violation de l'article 3 de la Convention, comme le montre notamment l'arrêt Peňaranda Soto c. Malte du 19 décembre 2017. Alors que les requérants dénonçaient un certain nombre d'éléments dégradés dans l'établissement (selon eux infesté de rats et de cafards, fournissant une nourriture et des vêtements peu adéquats, présentant de l'amiante dans les cellules - résidus de poussière calcaire selon le gouvernement - et tolérant le tabagisme passif), la Cour refuse de condamner l'État, en mettant en avant que l'intéressé avait été détenu dans une cellule individuelle, déverrouillée jusqu'à dix heures par jour, permettant donc de circuler librement dans les parties communes et d'accéder aux cours de promenade ${ }^{13}$.

Ce contrôle global des conditions de détention a été complété par des exigences plus précises posées par la Cour, afin de répondre aux besoins de certaines catégories de personnes privées de leur liberté. Ainsi en est-il de l'arrêt Korneykova et Korneykov c. Ukraine du 24 mars 2016, qui concerne une détenue et son bébé maintenus pendant plus de six mois dans de mauvaises conditions dans un centre de détention provisoire. Sur la base des photographies (fournies par le gouvernement), la Cour relève que si les intéressées ont pu séjourner dans une cellule lumineuse et en bon état, celle-ci ne possédait qu'un accès à l'eau froide de manière intermittente et pas d'eau chaude, et que l'insuffisance quantitative et qualitative de la nourriture sont corroborées par les déclarations de plusieurs autres détenues (la mère de la requérante ayant dû envoyer de nombreux colis de denrées alimentaires). Enfin, les promenades en plein air étaient insuffisantes ${ }^{14}$. Pour la Cour,

[...] l'effet cumulé de la malnutrition d'une mère allaitante, de conditions sanitaires et hygiéniques inadéquates pour la mère et son bébé, et de l'insuffisance des promenades en plein air, a dû être d'une intensité propre à engendrer une souffrance physique et une angoisse s'analysant en un traitement inhumain et dégradant pour la mère et l'enfant ${ }^{15}$

à l'origine d'une violation de l'article 3 .

Dans tous les cas, les dénonciations des mauvaises conditions de détention doivent reposer sur des preuves personnalisées, comme le souligne notamment l'arrêt Podeschi c. San-Marin du 13 avril 2017 dans lequel l'intéressé, qui dénonçait notamment l'impossibilité d'accéder à des équipements sanitaires adéquats, ne parvient pas à convaincre la Cour d'une violation de l'article 3. Les juges strasbourgeois rappellent en effet qu'ils attendent des requérants

[...] dans de telles situations qu'ils soumettent des informations détaillées et consistantes des faits dénoncés et qu'ils produisent, dans la mesure du possible, des preuves de leurs plaintes ${ }^{16}$

ce qui n'était pas le cas en l'espèce (les indications du requérant ayant par ailleurs été systématiquement réfutées par le gouvernement).

\section{La protection de la santé et de la qualité des soins}

Le fondateur arrêt Kudla (précité) fait peser sur les autorités pénitentiaires une prise en charge «adéquate» de la santé des personnes incarcérées ${ }^{17}$. Depuis une quinzaine d'années, la Cour s'est attachée à définir le contenu de cette obligation de soins, s'appliquant à toutes les pathologies et à tous les types de détenus et régimes de détention:

[...] l'exigence consistant à garantir la santé et le bien-être des détenus [s'incarne en] une obligation pour l'État de fournir à ces derniers les soins médicaux requis par leur état de santé ${ }^{18}$

Comme le rappelle l'arrêt Wenner c. Allemagne du $1^{\mathrm{er}}$ septembre 2016, la Cour cherche à faire respecter un principe d'équivalence des soins, qui doit garantir aux détenus un traitement médical dispensé dans des conditions comparables à celles dont bénéficie la population en milieu libre ${ }^{19}$, tout en rappelant que les États membres jouissent d'une certaine marge d'appréciation concernant le choix des soins à prodiguer aux détenus présentant des pathologies ${ }^{20}$. Cette marge d'appréciation renvoie notamment au lieu des soins, qui peuvent parfaitement être prodigués en prison, si l'état de santé du malade n'est pas incompatible avec la poursuite de son incarcération ${ }^{21}$, étant entendu que, lorsque

12. Cour EDH, 15 décembre 2016, Radzhab Magomedov c. Russie, nº 20933/08, $\$ 53$.

13. Cour EDH, 19 décembre 2017, Peňaranda Soto $c$. Malte, $\mathrm{n}^{\circ} 16680 / 14, \S 75$ sq.

14. Cour EDH, 24 mars 2016, Korneykova et Korneykov c. Ukraine, $\mathrm{n}^{\circ}$ 5666o/12, \$136 sq.

15. Ibid., $\$ 147$.

16. Cour EDH, 13 avril 2017, Podeschi c. San-Marin, no 66357/14, $\$ 112$.

17. Cour EDH, 26 octobre 200o, Kudla c. Pologne, $\$ 94$.

18. Cour EDH, 9 mai 2017, Eriomenco c. République de Moldova et Russie, no 42224/11, $\$ 53$.

19. Cour EDH, ${ }^{\text {er }}$ septembre 2016, Wenner c. Allemagne, $\mathrm{n}^{\circ} 62303 / 13, \S 66$.

20. Ibid., $\$ 58$.

21. Cour EDH, 18 février 2016, Rywin c. Pologne, n 6091/o6. Voir également Cour EDH, 2 mai 2017, Golubar c. Croatie, n² 21951/15; 7 septembre 2017, Mirzashvili c. Géorgie, n² 26657/07. 
le processus vital du détenu est engagé, il doit normalement pouvoir bénéficier d'une mesure d'élargissement, afin de vivre ses «derniers jours dans la dignité»" ${ }^{22}$. Qu'ils soient prodigués en détention ou dans un établissement civil ${ }^{23}$, les soins doivent toujours s'inscrire «dans une stratégie thérapeutique d'ensemble visant à traiter adéquatement les problèmes de santé» comme le rappelle notamment l'arrêt Yunusova et Yunusov c. Azerbaïdjan du 2 juin $2016^{24}$. Les détenus qui le nécessitent doivent donc pouvoir bénéficier de soins psychologiques ou psychiatriques ${ }^{25}$, d'une angiographie coronarienne rapide ${ }^{26}$, d'une chimiothérapie ${ }^{27}$, de soins dentaires ${ }^{28}$, de radiographies après une agression subie en détention ${ }^{29}$, d'un traitement rétroviral pour un détenu séropositif ${ }^{\circ \circ}$, de soins médicaux spécifiques pour un mineur de douze ans atteint de troubles mentaux et neurocomportementaux ${ }^{31}$, ou encore d'un traitement antituberculeux efficace ${ }^{32}$... Mais le respect de la santé en détention exige aussi que l'État fournisse un matelas ferme et un fauteuil roulant à une personne souffrant de graves problèmes neurologiques et orthopédiques ${ }^{33}$, un régime alimentaire adapté à des problèmes dentaires ${ }^{34}$, ou encore une canne ${ }^{35}$. Il convient néanmoins de souligner que toute dégradation de la santé en prison n'entraîne pas ipso facto la condamnation de l'État pour violation de l'article 3, comme le montre l'arrêt Cătălin Eugen Micu c. Roumanie du 5 juin 2016: souffrant d'une hépatite C, l'intéressé n'a pas apporté la preuve d'une contamination en prison. Par ailleurs, dès le diagnostic posé, il a pu recevoir l'assistance d'un médecin spécialiste et bénéficier des soins appropriés ${ }^{36}$. Enfin, tout défaut de soins entraînant un décès en détention nécessite de diligenter une enquête officielle et effective, qui soit de nature à permettre d'établir les causes de la mort, d'identifier les éventuels responsables de celle-ci et d'aboutir à leur punition ${ }^{37}$.
Au-delà de ces principes généraux, les juges strasbourgeois ont formulé des exigences médicales et sanitaires renforcées pour protéger les détenus les plus vulnérables. Ainsi, l'arrêt Korneykov et Korneykova (précité), qui insiste sur la particulière vulnérabilité des enfants détenus avec leur mère (et notamment des nouveau-nés), exige la surveillance étroite d'un spécialiste ${ }^{38}$. Cet arrêt rappelle également que le fait d'attacher une femme (qui ne manifestait aucune agressivité, ni velléité d'évasion) pendant la phase des contractions et immédiatement après son accouchement s'analyse en un traitement inhumain et dégradant ${ }^{39}$. L'arrêt Ábele c. Lettonie du 5 octobre 2017 relève également la particulière vulnérabilité des personnes sourdes qui, faute de disposer d'un appareil auditif performant, ne peuvent participer à des activités individuelles ou collectives pendant leur détention, générant un sentiment d' «isolement social» constitutif d'une violation de l'article $3^{40}$. Concernant la situation spécifique des détenus souffrant de troubles mentaux, la position de la Cour semble aujourd'hui claire: les autorités pénitentiaires doivent adopter à leur égard « une vigilance accrue», qui passe par un placement dans un établissement psychiatrique adapté ou dans une maison pénitentiaire dotée d'un pavillon psychiatrique spécialisé, sous peine de générer des situations de stress et d'angoisse, constitutives d'un traitement inhumain et dégradant ${ }^{41}$. Cette question est également au cœur de l'arrêt pilote $W$. D. c. Belgique du 6 septembre 2016, dans lequel la Cour retient une violation de l'article 5, $\$ 1$ de la Convention motivée par le constat général de l'inadaptation des annexes psychiatriques des prisons pour soigner les détenus condamnés déclarés pénalement irresponsables de leurs actes et souffrant de pathologies mentales graves. L'État belge est en conséquence invité à agir dans un délai de deux ans

22. Cour EDH, 28 novembre 2017, Dorneanu c. Roumanie, $\mathrm{n}^{\circ} 55089 / 13, \$ 97$.

23. La Cour étant particulièrement attentive au nombre et à la qualité des transferts entre le lieu de détention et l'établissement hospitalier, qui peuvent eux aussi générer une violation de l'article 3 s'ils sont trop nombreux et/ou s'effectuent dans des conditions de confort insatisfaisantes. Voir, inter alia, Cour EDH, 10 mai 2016, Topekhin c. Russie, $\mathrm{n}^{\circ} 78774 / 13 ; 13$ octobre 2016, Konovalchuk c. Ukraine, $\mathrm{n}^{\circ} 31928 / 15$.

24. Cour EDH, 2 juin 2016, Yunusova et Yunusov c. Azerbaïdjan, $\mathrm{n}^{\circ} 59620 / 14, \$ 142$; affaire qui présente une particularité procédurale, puisque les requérants (deux défenseurs des droits de l'homme et activistes réputés de la société civile) avaient obtenu de la Cour européenne des mesures provisoires (sur la base de l'article 39 du règlement) tendant à ce qu'on leur administre des soins adéquats en prison. Mesures provisoires non suivies d'effet comme le montre l'arrêt du 2 juin 2016. Dans le même sens, voir Cour EDH, 20 septembre 2016, Kondrulin c. Russie, n ${ }^{12987 / 15}$, concernant un requérant décédé d'un cancer en détention, faute de soins suffisants, malgré les mesures provisoires de la Cour demandant instamment à l'État de prodiguer des soins adéquats à l'intéressé.

25. Cour EDH, GC, 26 avril 2016, Murray c. Pays-Bas, n $10511 / 10$.

26. Cour EDH, 22 mars 2016, Kolesnikovich c. Russie, $\mathrm{n}^{\circ}$ 44694/13.

27. Cour EDH, $1^{\mathrm{er}}$ mars 2016, Andrey Lavrov c. Russie, n $\mathrm{n}^{\circ} 66252 / 14$

28. Cour EDH, 30 mai 2017, Apcov c. République de Moldavie et Russie, nº 13463/o7.

29. Cour EDH, 29 juin 2017, Dimcho Dimov c. Bulgarie ( $\left.n^{\circ} 2\right), \mathrm{n}^{\circ} 77248 / 12$.

30. Cour EDH, 12 janvier 2016, Khayletdinov c. Russie, $\mathrm{n}^{\circ} 2763 / 13$.

31. Cour EDH, GC, 3 mars 2016, Blokhin c. Russie, $\mathrm{n}^{\circ} 47152 / 06$

32. Cour EDH, 24 mai 2016, Makshakov c. Russie, $\mathrm{n}^{\circ}$ 52526/07.

33. Cour EDH, 21 mars 2017, Bujak c. Pologne, nº 686/12.

34. Cour EDH, 2 février 2016, Drăgan c. Roumanie, $\mathrm{n}^{\circ}$ 65158/o9.

35. Cour EDH, 9 juin 2016, Mekras c. Grèce, nº 12863/14.

36. Cour EDH, 5 juin 2016, Cătălin Eugen Micu c. Roumanie, n ${ }^{\circ} 55104 / 13$. Pour d'autres refus de reconnaitre une violation de l'article 3, voir Cour EDH, 21 juin 2016, Vasenin c. Russie, $\mathrm{n}^{\circ}$ 48023/06; 17 janvier 2017, Gengoux c. Belgique, $\mathrm{n}^{\circ}$ 76512/11; 4 décembre 2017, Kitiashvili c. Géorgie, $\mathrm{n}^{\circ} 37747 / 08$.

37. Voir, inter alia, Cour EDH, 14 février 2017, Karakhanyan c. Russie, $\mathrm{n}^{\circ}$ 24421/11.

38. Cour EDH, 24 mars 2016, Korneykova et Korneykov c. Ukraine, $\$ 152$.

39. Ibid., \$112 sq.

40. Cour EDH, 5 octobre 2017, Ābele c. Lettonie, $\$ 69$ et 70 .

41. Cour EDH, 9 janvier 2018, Kadusic c. Suisse, $\mathrm{n}^{\mathrm{o}}$ 43977/13. Sur cette question, voir également Cour EDH, $1^{\mathrm{er}}$ juin 2017, Rooman c. Belgique, $\mathrm{n}^{\mathrm{o}} 18 \mathrm{0} 52 / 11$. 
[...] afin de réduire le nombre de personnes ayant commis des crimes ou des délits souffrant de troubles mentaux qui sont internées, sans encadrement thérapeutique adapté, au sein des ailes psychiatriques des prisons notamment en redéfinissant $[\ldots]$ les critères justifiant une mesure d'internement ${ }^{42}$.

\section{Le contrôle des violences en détention}

S'il existe aux yeux de la Cour un «niveau inévitable de souffrance inhérent à la détention » ${ }^{43}$, les personnes privées de leur liberté ne peuvent en aucun cas être traitées «comme des objets aux mains de la puissance publique», ni vivre "pendant toute la durée de leur détention dans un lieu de "non-droit" où les garanties les plus élémentaires [sont] suspendues ${ }^{44}$. Le travail jurisprudentiel de la Cour permet de rappeler quels sont les comportements constitutifs de violences inacceptables dans les prisons d'États démocratiques et à ce titre pouvant être qualifiés de traitements inhumains ou dégradants (voire de torture). Cette mission s'exerce tant à l'égard des violences commises entre détenus, que celles commises par le personnel de surveillance. Dans tous les cas, les allégations de mauvais traitements doivent être étayées par des éléments de preuve adéquats, «au-delà de tout doute raisonnable», résultant d'un faisceau d'indices, ou de présomptions non réfutées, suffisamment graves, précis et concordants ${ }^{45}$. Si le recours à la force par le personnel de surveillance peut être parfois nécessaire, la Cour veille à ce que cette utilisation soit toujours légitime et proportionnée. Ainsi, l'infliction de coups et l'utilisation de matraques en caoutchouc lors d'une opération de fouille de cellule alors que le détenu concerné n'avait pas posé de problèmes particuliers est constitutive d'un traitement violant l'article 3 de la Convention $^{46}$. De même l'utilisation de grenades pour réprimer une émeute dans un établissement pénitentiaire (et ce alors que rien ne permettait d'établir que les requérants avaient activement pris part à l'émeute et attaqué les forces de l'ordre) ne constitue pas un usage de la force rendu absolument nécessaire par le comportement des intéressés ${ }^{47}$. Tout comme des coups violents visant à forcer un détenu à avouer un meurtre, accompagnés de plusieurs placements dans une cellule d'isolement et de menaces de «malheurs" qui allaient arriver à sa petite amie et à sa sœur, constituent une violation de l'article $3^{48}$. Certaines violences graves peuvent même être qualifiées de torture comme le montre l'arrêt Cirino et Renne c. Italie du 26 octobre $2017^{49}$ : soumis, afin de les punir, à des violences physiques, répétées à toute heure du jour ou de la nuit pendant plusieurs jours d'affilée, les requérants ont dû également subir des privations matérielles extrêmes (rationnement d'eau et de nourriture, absence de sanitaires, de literie et de chauffage appropriés) et une humiliation supplémentaire causée par l'obligation de rester nus pendant plusieurs jours. Bien évidemment, l'utilisation de la force à l'encontre des détenus ne doit jamais conduire au décès de ceuxci. Ainsi une mesure de compression effectuée par des gardiens se croyant menacés, et qui conduit à la mort d'un détenu souffrant de troubles psychiatriques, constitue une violation de l'article 2 de la Convention (arrêt Tekın et Arslan c. Belgique du 5 septembre 2017). Ce même arrêt permet à la Cour de rappeler qu'il est nécessaire que les agents pénitentiaires disposent d'instructions claires et adéquates relatives aux techniques manuelles de maîtrise de détenus ${ }^{50}$.

Par ailleurs, lorsqu'une personne se trouvant entre les mains d'agents de l'État invoque des allégations de mauvais traitements contraires à l'article 3 (ou à l'article 2 lorsque ces mauvais traitements ont occasionné un décès), les autorités doivent aussitôt diligenter une enquête approfondie, effective et adéquate, c'est-à-dire apte à conduire l'établissement des faits, permettre de vérifier si le recours à la force était justifié en l'espèce, identifier et - le cas échéant - sanctionner le ou les coupables En outre, une indemnité doit être accordée à la victime de ces sévices, ou tout du moins celle-ci doit avoir la possibilité de solliciter et d'obtenir une réparation suffisante pour le préjudice causé (Milić et Nikezić c. Monténégro). Par ailleurs, les agents inculpés d'infractions impliquant des mauvais traitements doivent être suspendus de leurs fonctions pendant l'instruction ou le procès et démis en cas de condamnation (Azzolina et autres $c$. Italie). Ainsi ne respecte pas les exigences procédurales une enquête diligentée après des mauvais traitements infligés par des gardiens, sans approfondir les différentes versions, ni contester la version des gardiens qui justifiaient l'usage de la force par un refus d'obtempérer du requérant ${ }^{51}$. Manque également d'effectivité une enquête sur des mauvais traitements allégués ne reposant que sur une vidéo de six minutes, alors que le détenu avait pu prouver que la fouille contestée avait duré trente minutes ${ }^{52}$, ou encore une enquête n'ayant abouti à entendre les agents

42. Cour EDH, 6 septembre 2016, W. D. c. Belgique, $\mathrm{n}^{\circ} 73548 / 13, \S 170$.

43. Voir, inter alia, Cour EDH, 3 octobre 2017, Alexandru Enache c. Roumanie, $\$ 47$.

44. Cour EDH, 26 octobre 2017, Azzolina et autres c. Italie, $n^{\circ} 28923 / 09$ et $67599 / 10, \$ 134$.

45. Cour EDH, 12 décembre 2016, Idalov c. Russie ( $\left.n^{\circ} 2\right), \mathrm{n}^{\circ} 41858 / 08, \$ 83$.

46. Cour EDH, 28 avril 2015, Milić et Nikezić c. Monténégro, no 54999/10 et 10609/11.

47. Cour EDH, 22 mars 2016, Kars et autres $c$. Turquie, $\mathrm{n}^{\circ}$ 66568/o9, $\$ 85$.

48. Cour EDH, 14 septembre 2009, Matevosyan c. Arménie, $\mathrm{n}^{\circ}$ 52316/o9. Sur des violences visant à faire avouer un détenu, voir également Cour EDH, 12 janvier 2016, Morgoci c. République de Moldova, $\mathrm{n}^{\circ}$ 13421/o6; 5 octobre 2017, Ostrovenecs c. Lettonie, $\mathrm{n}^{\circ} 36043 / 13$

49. Cour EDH, 26 octobre 2017, Cirino et Renne c. Italie, $\mathrm{n}^{\circ} 2539 / 13$ et 4705/13.

50. Cour EDH, 5 septembre 2017, Tekin et Arslan c. Belgique, $\mathrm{n}^{\circ} 37795 / 13, \$ 92$.

51. Cour EDH, 7 novembre 2017, Bambayev c. Russie, $\mathrm{n}^{\circ}$ 19816/o9, $\$ 42$.

52. Cour EDH, 15 mars 2016, Ciorap c. République de Moldova ( $\left.n^{\circ} 5\right), \mathrm{n}^{\circ}$ 7232/o7. 
suspectés que plus d'un an après les faits, ce qui a accru les risques de collusion entre les intéressés ${ }^{53}$.

Les violences en prison sont également des violences que s'infligent les détenus soit entre eux, soit eux-mêmes et dont la forme la plus extrême est le suicide. À cet égard, la Cour exige des États la mise en œuvre de mesures préventives efficaces. L'arrêt Isenc c. France du 4 février 2016 démontre notamment, qu'aux yeux des juges strasbourgeois, «il ne saurait être question de réduire la prise en charge d'une personne détenue en détresse aux seules mesures de surveillance ${ }^{54}$. Détenu «primaire» et signalé dès le début de son incarcération comme présentant des tendances suicidaires ${ }^{55}$, le fils du requérant n'a, semblet-il, fait l'objet d'aucune surveillance médicale spécifique, alors qu'un contrôle de santé lors de son admission aurait constitué «une mesure de précaution minimale ${ }^{56}$. La grande vulnérabilité de certains détenus (tels que les mineurs) nécessite par ailleurs des mesures de protection particulières. Ainsi, face à des violences sexuelles commises par des codétenus (sans que les autorités aient été préalablement informées du risque de survenance de ces sévices), la Cour conclut que les autorités n'ont pas failli

[...] à leur obligation positive de protéger l'intégrité physique du requérant dans le cadre de leur devoir consistant à surveiller les personnes privées de liberté et à empêcher qu'il ne soit porté atteinte à leur intégrité physique ${ }^{57}$.

En effet, l'administration pénitentiaire a ouvert une enquête interne dès qu'elle a eu connaissance des faits, la victime a immédiatement été transférée vers un hôpital pour y subir un examen médical, les déclarations de tous les protagonistes ont été recueillies et l'agresseur s'est vu infliger une sanction disciplinaire. Par la suite, l'administration pénitentiaire a avisé le procureur de la République de l'incident, ce dernier a diligenté une enquête conduisant à l'ouverture d'une procédure pénale à l'encontre de l'agresseur et, à l'issue du procès, celui-ci a été condamné à huit ans et neuf mois d'emprisonnement.

\section{Le respect de la vie privée et familiale et des relations avec l'extérieur}

Même si les contraintes maximales de sécurité limitent fortement en pratique la mise en œuvre de cette disposition, les détenus jouissent bien de la protection de l'article 8 de la Convention de 1950 qui protège tout à la fois la vie privée, la vie familiale et les correspondances.

Le contentieux de l'article 8 (souvent lié à l'article 3 en la matière) appliqué à la situation spécifique des détenus a permis à la Cour de poser depuis plusieurs années des règles claires concernant l'utilisation et le déroulement des fouilles en prison, quelles que soient leurs formes et modalités pratiques. L'arrêt Dejnek $c$. Pologne du $1^{\mathrm{er}}$ juin 2017 permet ainsi à la Cour de rappeler que, si les fouilles à corps peuvent dans certains cas s'avérer nécessaires "pour garantir la sécurité à l'intérieur de la prison ou pour prévenir des troubles ou des infractions ${ }^{58}$, celles-ci doivent toujours s'effectuer de manière appropriée, dans des conditions respectueuses de la dignité humaine, être motivées par des raisons légitimes ${ }^{59}$ et ne doivent pas être prévues à un rythme excessif, même pour des détenus dangereux soumis à un régime de haute sécurité ${ }^{60}$. Ainsi, les fouilles intégrales ne peuvent pas constituer une pratique systématique et routinière lors des fouilles de cellules ${ }^{61}$. En l'espèce, elles étaient mises en œuvre en application des dispositions pertinentes du Code de l'exécution des peines, pour des motifs justifiés (en raison d'une suspicion de trafic, puisque de l'argent d'une provenance inconnue et de la drogue avaient été trouvés dans les vêtements du requérant; celui-ci avait également tenté de faire passer des messages à sa famille et il avait enfin été classé détenu dangereux), leur déroulement a respecté la dignité du détenu. Elles ne peuvent en conséquence être considérées comme constitutives d'un traitement humiliant ou dégradant $^{62}$. Par ailleurs, si des systèmes de surveillance vidéo des espaces communs des lieux de détention, et même des cellules peuvent être mis en place, cette possibilité doit toujours être "prévue par la loi » ${ }^{63}$. L'arrêt Biržietis c. Lituanie du 14 juin 2016 rappelle par ailleurs que le respect du droit à la vie privée des détenus protège leur droit à l'identité personnelle, qui inclut notamment le choix de se laisser pousser la barbe, «motivé par la volonté d'exercer son droit d'exprimer sa personnalité et son identité ${ }^{64}$. L'interdiction totale de la barbe prévue par le règlement intérieur (justifiée pour des raisons d'hygiène ou par la nécessité d'identifier les détenus) ne renvoie selon la Cour à aucun «besoin social impérieux» ${ }^{65}$.

Privés de leur liberté, soit en attente de jugement, soit pour purger une peine, les détenus sont toujours considérés

53. Cour EDH, 19 avril 2016, Gheorghe Dima c. Roumanie, $\mathrm{n}^{\circ}$ 2770/o9. Sur des défauts d'enquête, voir également Cour EDH, 28 avril 2016, Balajevs c. Lettonie, $\mathrm{n}^{\circ} 8347 / 07 ; 1^{\text {er }}$ décembre 2016, Trapeznikova et autres c. Russie, $\mathrm{n}^{\circ}$ 45115/o9; 16 mai 2017, Romanescu c. Roumanie, $\mathrm{n}^{\circ} 78375 / 11$.

54. Cour EDH, 4 février 2016, Isenc c. France, $\mathrm{n}^{\circ} 58828 / 13, \S 44$.

55. Ibid., $\$ 49$.

56. Ibid., $\$ 45$.

57. Cour EDH, 13 septembre 2016, A. Ş. c. Turquie, $\mathrm{n}^{\circ} 58271 / 10, \S 80$.

58. Cour EDH, $1^{\text {er }}$ juin 2017 , Dejnek c. Pologne, $n^{\circ} 9635 / 13, \$ 60$ et 75 .

59. Ibid., $\$ 60$.

60. Cour EDH, 21 mars 2017, Michat Korgul c. Pologne, $\mathrm{n}^{\circ} 36140 / 11$.

61. Cour EDH, $1^{\mathrm{er}}$ juin 2017, Dejnek c. Pologne, $\$ 75$. Voir également Cour EDH, 16 février 2016, Paluch c. Pologne, $\mathrm{n}^{\circ} 57292 / 12$ et Świderski c. Pologne, $\mathrm{n}^{\circ} 5532 / 10$.

62. Cour EDH, $1^{\text {er }}$ juin 2017, Dejnek c. Pologne, $\$ 60-61$.

63. Cour EDH, 6 décembre 2016, Vasilică Mocanu c. Roumanie, no 43545/13, 33 sq.

64. Cour EDH, 14 juin 2016, Biržietis c. Lituanie, $\mathrm{n}^{\circ}$ 49304/o9, $\$ 33$.

65. Ibid., $\$ 58$. 
par la Cour européenne comme des membres à part entière de la société, ce qui exige notamment qu'ils possèdent des perspectives réelles d'élargissement dans le cadre de peines toujours "compressibles" ${ }^{66}$. Ainsi, dans ses arrêts T. P. et A. T. c. Hongrie du 4 octobre 2016, la Cour juge trop longue une période d'attente de quarante ans avant qu'un détenu condamné à une peine perpétuelle puisse pour la première fois espérer qu'une mesure de clémence soit envisagée dans son cas. La législation interne n'est dès lors pas compatible avec l'article 3 de la Convention ${ }^{67}$. Par contre dans l'arrêt Hutchinson c. Royaume-Uni du 17 janvier $2017^{68}$, la Cour européenne des droits de l'homme, à la majorité, conclut à la non-violation de l'article $3^{69}$ en relevant que les juridictions britanniques ont clarifié les dispositions du droit interne concernant le réexamen des peines perpétuelles, tout spécialement tant la portée, que les motifs et les modalités du réexamen par le ministre, ainsi que l'obligation pour celui-ci de libérer tout détenu condamné à une peine de perpétuité réelle dont le maintien en détention n'est plus justifiable. L'arrêt Hutchinson permet à la Cour de rappeler l'impact décisif de la loi anglaise de 1998 sur les droits de l'homme (Human Rights Act).

L'obligatoire retour à la vie libre explique l'extrême importance du maintien des liens avec l'extérieur, qui s'expriment notamment à travers les visites, dont le régime doit être fixé par la loi avec suffisamment de précision ${ }^{70}$. Si la Cour rappelle régulièrement que «la détention, comme toute autre mesure privative de liberté, entraîne par nature des restrictions à la vie privée et familiale», elle ajoute toutefois qu'il «est cependant essentiel au respect de la vie familiale que l'administration pénitentiaire autorise le détenu, et l'aide au besoin, à maintenir le contact avec sa famille proche $»^{71}$. En conséquence,

[...] les autorités nationales ont l'obligation de prévenir la rupture des liens familiaux et doivent assurer aux prisonniers un niveau de contact avec leur famille raisonnablement satisfaisant, avec des visites organisées aussi fréquemment que possible, et dans des conditions aussi normales que possible $^{72}$

Si la Cour se refuse à considérer qu'il existe un consensus européen concernant la fréquence de ces visites, l'arrêt
Kalda c. Estonie du 19 janvier 2016 souligne cependant qu' « une visite par mois au moins constitue la fréquence minimale généralement admise» (notamment pour les détenus soumis aux régimes de détention les plus stricts ${ }^{73}$ ). Il va de soi qu'aux yeux de la Cour l'action des autorités pénitentiaires vise à faciliter et non restreindre leur exercice comme le relève l'arrêt Vidish c. Russie du 15 mars 2016 dans lequel l'État est condamné en raison de l'existence d'une «taxe de visite» dont devaient s'acquitter tous les visiteurs (d'un montant de 320 roubles - soit environ 9 euros - par jour et par visiteur, elle était sensée couvrir des frais de maintenance et d'entretien de la salle de visites et contribuer au paiement des salaires des agents pénitentiaires) ${ }^{74}$. De même, des visites familiales se déroulant avec la présence continue d'un gardien imposant l'utilisation du russe et non de l'allemand (langue maternelle du détenu et de ses visiteurs) sous peine d'annulation de la visite constituent une violation de la Convention (Mozer c. Moldavie et Russie). La jurisprudence très protectrice de la Cour ne va toutefois pas jusqu'à poser un droit au regroupement familial des personnes incarcérées, car selon elle «la Convention n'accorde pas aux détenus le droit de choisir leur lieu de détention, la séparation et l'éloignement du détenu de sa famille constituant des conséquences inévitables de la détention ${ }^{75}$. La nécessité du maintien d'un lien effectif avec l'extérieur nécessite même parfois que le prisonnier puisse sortir de l'établissement dans des circonstances particulières, assister par exemple à l'inhumation d'un proche ${ }^{76}$. Le maintien (ou la création) des liens familiaux peut aussi s'exprimer par le mariage (ou le remariage), puisque l'article 12 de la Convention "protège le droit fondamental pour tout homme et toute femme de se marier et de fonder une famille». Ce droit

[...] est mis en œuvre par les lois nationales des États membres, mais les limitations introduites ne peuvent le restreindre ou le réduire à un point tel que l'essence de ce droit n'en soit altérée ${ }^{77}$.

Les relations avec l'extérieur passent également par le courrier. En particulier, les échanges avec l'avocat doivent bénéficier de garanties appropriées permettant de préserver la confidentialité du contenu de cette

66. Cour EDH, GC, 24 janvier 2017, Khamtokhu et Aksenchikc. Russie, no 60367/o8 et 961/11.

67. Cour EDH, 4 octobre 2016, T. P. et A. T. c. Hongrie, n $37871 / 14$ et 73986/14. N'est pas davantage compatible avec la Convention européenne des droits de l'homme une législation qui réserve au président de la République le dernier mot pour décider si le maintien en prison est justifié. La loi ne prévoit par ailleurs pas de délai au président pour se prononcer sur la demande de mesures de clémence et ne l'oblige pas à motiver sa décision, même si elle s'écarte de la recommandation formulée par la commission des grâces (T. P. et A. T. c. Hongrie). Voir également Cour EDH, 23 mai 2017, Matiošaitis et autres c. Lituanie, no 22662/13, 51059/13, 58823/13, 59692/13, 59700/13, 60115/13, 69425/13 et 72824/13.

68. Cour EDH, GC, 17 janvier 2017, Hutchinson c. Royaume-Uni, nº 57592/o8.

69. Contrairement à la solution qui avait été retenue dans l'arrêt Vinter et autres c. Royaume-Uni (GC) du 9 juillet 2013, nº 66069/o9, 130/10 et 3896/10.

70. Cour EDH, 2 mars 2017, Moroz c. Ukraine, $\mathrm{n}^{\circ}$ 5187/o7. Voir également Cour EDH, 30 mai 2017, Vardanean c. République de Moldavie et Russie, $\mathrm{n}^{\circ} 22200 / 10$.

71. Voir, inter alia, Cour EDH, GC, 23 février 2016, Mozer c. Moldavie et Russie, nº 11138/10, $\$ 190$.

72. Cour EDH, 7 mars 2017, Polyakova et autres c. Russie, no $35090 / 09,35845 / 11,45694 / 13$ et 59747/14, $\$ 89$.

73. Cour EDH, 19 janvier 2016, Kalda c. Estonie, $\mathrm{n}^{\circ}$ 17429/10, $\$ 135$.

74. Cour EDH, 15 mars 2016, Vidish c. Russie, $\mathrm{n}^{\circ} 53120 / 08$.

75. Cour EDH, 2 mars 2017, Labaca Larrea et autres c. France, no $56710 / 13,56727 / 13,57412 / 13, \$ 42$.

76. Cour EDH, 6 décembre 2016, Kanalas c. Roumanie, $\mathrm{n}^{\circ}$ 420323/14. Voir également 16 mai 2017, Pakhtusov c. Russie, $\mathrm{n}^{\circ} 11800 / 10 ;$ Sokolov c. Russie, 21 novembre 2017, n 63392/o9.

77. Cour EDH, 8 décembre 2016, Chernetskiy c. Ukraine, ${ }^{\circ} 44316 / 07, \$ 28$. En l'espèce, le requérant ne pouvait se remarier, en raison du refus opposé par les autorités de finaliser la procédure de son divorce et de lui fournir en prison son certificat de divorce $(\$ 31)$. 
correspondance $^{78}$, de même que les communications téléphoniques qui doivent être organisées selon des modalités connues du détenu ${ }^{79}$. Les jeunes placés en établissement éducatif fermé non reconnus coupables d'infractions pénales ne peuvent quant à eux se voir imposer une restriction indifférenciée de leur droit à la correspondance. Une telle mesure serait en effet contraire au but même de ce type de structure, qui est de prodiguer une éducation et soutenir ces personnes dans leur réinsertion sociale ${ }^{80}$. Sensible à la place prise par les nouvelles technologies de l'information, la Cour de Strasbourg a même entrouvert l'accès à Internet pour les personnes incarcérées dans son arrêt du 19 janvier 2016, Kalda c. Estonie: si l'article 10 de la Convention de 1950 ne peut être considéré comme la source d'un véritable droit d'accès à Internet pour les détenus ${ }^{81}$, les juges strasbourgeois exigent toutefois que toute ingérence dans le droit des détenus à recevoir des informations par ce support soit "prévue par la loi ${ }^{82}$.

\section{L'existence de voies de recours effectives}

L'amélioration des droits des personnes incarcérées passe également par l'existence de voies de recours, qui permettent de dénoncer devant une juridiction (ou une instance comparable disposant d'un statut d'indépendance et de pouvoir de décision étendus) toute violation de leurs droits. Cet accès au recours (qui doit pouvoir se dérouler sans «craindre de souffrir de conséquences négatives en raison de cette action ${ }^{83}$ ) constitue d'ailleurs une exigence explicite de la Convention européenne des droits de l'homme, qui précise dans son article 13 que

Toute personne dont les droits et libertés reconnus dans la présente Convention ont été violés, a droit à l'octroi d'un recours effectif devant une instance nationale, alors même que la violation aurait été commise par des personnes agissant dans l'exercice de leurs fonctions officielles.

Le contrôle de la dignité des conditions matérielles de détention opéré par la Cour de Strasbourg est ainsi complété par l'exigence de voies de recours effectives en droit interne, qui permettent de faire constater des conditions de détention inadéquates, et de réparer les dommages causés, comme le démontrent par exemple les arrêts du 21 mars 2017, Kargashin et autres c. Russie et Mozharov et autres c. Russie dans lesquels les ressortissants se plaignaient (pendant des durées de neuf mois à onze ans) d'une situation de surpopulation carcérale, couplée à l'obligation de partager un lit avec d'autres détenus et de dormir à tour de rôle, une détention dans des bâtiments froids infestés de rats, souris, punaises et vermine, où manquait l'air frais, où la nourriture était de piètre qualité et l'eau chaude rare ou absente. L'absence de recours effectif leur permettant de se plaindre de ces conditions de détention a constitué à leur égard une violation de l'article $13^{84}$. Ces voies de recours doivent également permettre de dénoncer d'autres situations, telles que l'impossibilité d'accéder à un traitement médical adéquat ${ }^{85}$, ou encore des conditions inhumaines et dégradantes de transport et de détention au tribunal lors des audiences, en raison du surpeuplement et du manque d'aération dans les fourgonnettes, les compartiments de train et les cellules du tribunal ${ }^{86}$. L'arrêt Valentin Baştovoi c. République de Moldova du 28 novembre 2017 est également révélateur des méthodes employées par la Cour: condamnant l'État en raison de mauvaises conditions de détention constitutives d'une violation de l'article 3 , elle retient également une violation de l'article 13, puisqu'il n'existe pas en droit national de recours permettant de mettre fin à de telles situations. Mais la Cour ajoute surtout qu'elle a déjà eu l'occasion de se pencher à de multiples reprises sur la question de savoir s'il existait dans cet État des voies de recours effectives relativement à des allégations de mauvaises conditions de détention et qu'elle a à chaque fois estimé que les recours invoqués par le gouvernement étaient ineffectifs à l'égard des personnes qui continuaient à être détenues dans des conditions incompatibles avec l'article 3 de la Convention ${ }^{87}$.

Le constat effectué par l'arrêt Baştovoi démontre que ce contentieux, loin d'être circonscrit à des cas particuliers, renvoie au contraire bien souvent à des situations concernant de manière comparable de nombreux requérants, constitutives d'un "problème structurel», selon le vocabulaire employé par la Cour. Ainsi en est-il de l'arrêt Rezmives et autres $c$. Roumanie du 25 avril 2017 dans lequel les requérants se sont plaints du surpeuplement des cellules, mais aussi du manque et de l'insalubrité des installations sanitaires, de l'hygiène déplorable, de trop courtes promenades, de lumière et de ventilation

78. Cour EDH, 13 décembre 2016, Eylem Kaya c. Turquie, nº 26623/o7.

79. Cour EDH, 19 juillet 2017, Lebois c. Bulgarie, $\mathrm{n}^{\circ} 67482 / 14$.

80. Cour EDH, 19 mai 2016, D. L. c. Bulgarie, $\mathrm{n}^{\circ} 7472 / 14$.

81. Cour EDH, 19 janvier 2016, Kalda c. Estonie, $\mathrm{n}^{\circ}$ 17429/10, $\$ 45$.

82. Voir également Cour EDH, 17 janvier 2017, Jankovskis c. Lituanie, $\mathrm{n}^{\circ}$ 21575/o8.

83. Cour EDH, 21 juillet 2016, Shahanov et Palfreeman c. Bulgarie, $\mathrm{n}^{\circ} 35365 / 12$ et $69125 / 12, \$ 64$.

84. Cour EDH, 21 mars 2017, Kargashin et autres c. Russie, n ${ }^{\circ}$ 66757/14 sq. et Mozharov et autres c. Russie, $\mathrm{n}^{\circ} 16401 / 12$ sq.

85. Voir, inter alia, Cour EDH, 12 janvier 2016, Khayletdinov c. Russie, $\mathrm{n}^{\circ}$ 2763/13; 4 octobre 2016, Klimov c. Russie, $\mathrm{n}^{\circ} 54436 / 14$.

86. Cour EDH, 12 décembre 2016, Idalov c. Russie ( $\left.n^{\circ} 2\right), \mathrm{n}^{\circ} 41858 / 08$.

87. Cour EDH, 28 novembre 2017, Valentin Bastovoi c. République de Moldova, $\mathrm{n}^{\circ} 40614 / 14, \$ 17$. Sur d'autres violations combinées des articles 3 et 13, voir, inter alia, Cour EDH, 26 janvier 2016, Patrikis et autres c. Grèce, n ${ }^{\circ}$ 50622/13; 28 janvier 2016, Konstantinopoulos et autres c. Grèce, $\mathrm{n}^{\circ}$ 69781/13; 22 mars 2016, Butrin c. Russie, $\mathrm{n}^{\circ}$ 16179/14; 29 mars 2016, Okolisan c. République de Moldova, nº 33200/11; 5 juillet 2016, Bandur c. Hongrie, $\mathrm{n}^{\circ}$ 50130/12; 6 octobre 2016, Kalandia c. Grèce, $\mathrm{n}^{\circ} 48684 / 15 ; 19$ janvier 2017, Singh et autres c. Grèce, $\mathrm{n}^{\circ} 60041 / 13 ; 9$ février 2017, Igbo et autres c. Grèce, nº $60042 / 13$. 
insatisfaisantes, de la mauvaise qualité de la nourriture, de la vétusté du matériel fourni, ainsi que de la présence de nuisibles ${ }^{88}$. Le caractère connu de ces dénonciations ${ }^{89}$ aboutit à ce que la Cour européenne fasse de l'arrêt du 25 avril 2017 un «arrêt pilote » : il s'agit d'indiquer à l'État quelles mesures il doit prendre, dans un délai préfixé, afin d'éviter la condamnation, ainsi que de nouvelles saisines et condamnations dans des affaires comparables ${ }^{90}$. Très concrètement, la Roumanie a six mois pour mettre en œuvre des mesures alternatives à la détention provisoire, pour renforcer l'accès aux mesures de libération conditionnelle et de semi-liberté, pour créer un système de probation efficace, pour rénover les lieux de détention existants, et pour mettre en place une voie de recours préventive permettant au juge de surveillance de l'exécution et aux tribunaux de mettre fin à la situation contraire à l'article 3 de la Convention, ainsi qu'une voie de recours compensatoire permettant d'obtenir une indemnisation adéquate en cas de mauvaises conditions matérielles de détention ${ }^{91}$. L'arrêt Domján c. Hongrie du 23 novembre $2017^{92}$ a quant à lui permis à la Cour de vérifier les suites de l'arrêt pilote Varga et autres c. Hongrie du 10 mars $2015^{93}$ : dans cet arrêt pilote, la Cour avait constaté l'existence d'un problème général résultant du mauvais fonctionnement du système pénitentiaire hongrois, générant des violations des articles 3 et 13 de la Convention. Elle avait en conséquence demandé la mise en place de recours préventifs et compensatoires, pouvant être exercés en cas d'allégations de violations de l'article 3 fondées sur des conditions de détention inhumaines et / ou dégradantes. L'arrêt Domján c. Hongrie constate que le 25 octobre 2016 le Parlement hongrois a bien adopté une loi (entrée en vigueur le $1^{\text {er }}$ janvier 2017) établissant un ensemble de recours, de nature tant préventive que compensatoire, qui garantissent en principe un véritable redressement pour les violations de la Convention découlant de la surpopulation carcérale et d'autres conditions de détention inappropriées en Hongrie. M. Domján et tous les autres détenus placés dans une situation comparable doivent donc désormais utiliser les recours introduits par cette nouvelle loi, les requêtes pendantes devant la Cour européenne des droits de l'homme étant par contre rejetées en raison de leur caractère prématuré.

Au-delà de l'exigence générale posée par l'article 13, la Convention de 1950 protège aussi le droit à la sûreté dans son article 5, qui exige qu'un juge contrôle le caractère légal de la privation de liberté (qu'elle se déroule avant ou après un jugement), et que le détenu obtienne le cas échéant une réparation en cas de violation de cette disposition ${ }^{94}$. Le contrôle de l'utilisation légale de la privation de liberté ${ }^{95}$ permet notamment à la Cour de s'assurer que le recours à la prison n'est pas dévoyé et correspond bien à des finalités conformes aux buts et principes de la Convention de 1950, justifiées par des motifs «pertinents et suffisants ${ }^{96}$. Ainsi, l'arrêt Merabishvili c. Géorgie du 14 juin 2016 rappelle que la prison ne peut être une technique de contrôle des opposants politiques : ayant occupé les fonctions de Premier ministre au cours de l'année 2012, et devenu l'un des leaders de l'opposition depuis l'arrivée de la coalition Rêve géorgien aux élections législatives d'octobre 2012, le requérant a selon la Cour certes été détenu pour des raisons pénales, mais surtout afin d'éviter qu'il ne se présente aux élections présidentielles de 2013. Or, pour les juges strasbourgeois, la perspective d'une détention ne peut être utilisée comme le moyen d'exercer une pression morale sur un accusé ${ }^{97}$. Dans le même sens, l'arrestation et la condamnation à cinq jours de « détention administrative » de participants à une marche de protestation pacifique organisée par des opposants politiques (même non autorisée) n'est pas conforme aux exigences conventionnelles. Même si la sanction a été prise conformément aux prescriptions législatives en vigueur, elle ne correspond en effet à "aucun besoin social impérieux". Bien davantage, de telles mesures risquent également de

88. Cour EDH, 25 avril 2017, Rezmives et autres c. Roumanie, $\mathrm{n}^{\circ} 61467 / 12,39516 / 13,48231 / 13$ et 68191/13

89. Puisque déjà identifié par la Cour dans son arrêt du 24 juillet 2012, Iacov Stanciu c. Roumanie, n $35972 / 05$ et ayant entraîné quatre-vingt-treize condamnations de la Roumanie entre 2007 et 2012.

90. Technique que la Cour avait déjà eu l'occasion d'utiliser concernant les conditions de détention indignes en Italie dans son arrêt pilote Torreggiani et autres c. Italie du 8 janvier 2013 ( $\mathrm{n}^{\circ}$ 43517/o9, 46882/o9, 55400/o9, 57875/o9, 61535/o9, 35315/10 et 37818/10). Voir J.-M. Larralde, «Chronique de jurisprudence de la Cour européenne des droits de l'homme relative aux prisons 2014-2015", Cahiers de la recherche sur les droits fondamentaux, $\mathrm{n}^{\circ} 14,2016$, p. 150

91. L'arrêt est également l'occasion pour la Cour de rappeler que la construction de nouvelles places de prison n'est pas une solution durable pour remédier aux situations de surpeuplement carcéral.

92. Cour EDH, 23 novembre 2017, Domján c. Hongrie, ${ }^{\circ} \mathrm{n}^{\circ}$ 5433/17.

93. Cour EDH, 10 mars 2015, Varga et autres c. Hongrie, $\mathrm{n}^{\circ}$ 14097/12. Voir J.-M. Larralde, "Chronique... ", p. 151.

94. Article $5, \S 3$ de la Convention: «Toute personne arrêtée ou détenue [...] doit être aussitôt traduite devant un juge ou un autre magistrat habilité par la loi à exercer des fonctions judiciaires et a le droit d'être jugée dans un délai raisonnable, ou libérée pendant la procédure [...]»; article 5 , $\$ 4$ de la Convention: «Toute personne privée de sa liberté par arrestation ou détention a le droit d'introduire un recours devant un tribunal afin qu'il statue à bref délai sur la légalité de sa détention et ordonne sa libération si la détention est illégale».

95. Selon l'article $5, \S 1$ de la Convention, la privation de liberté d'une personne n'est possible, selon les voies légales, qu'après condamnation par un tribunal compétent (a), ou après une arrestation ou une détention régulière pour insoumission à une ordonnance rendue conformément à la loi par un tribunal (b), ou en vue de la conduire devant une autorité judiciaire en cas de raisons plausibles de soupçonner qu'une infraction a été commise (c), ou pour détenir un mineur en vue de son éducation surveillée ou pour le traduire devant une autorité compétente (d), ou pour détenir une personne susceptible de propager une maladie contagieuse, un aliéné, un alcoolique, un toxicomane, un vagabond (e), ou pour empêcher une personne de pénétrer irrégulièrement sur le territoire ou contre une personne en vue de son expulsion ou extradition (f)

96. Cour EDH, 21 mars 2017, Porowski c. Pologne, $\mathrm{n}^{\circ}$ 34458/03, $\$ 137$ sq.

97. Cour EDH, 14 juin 2016, Merabishvili c. Géorgie, $\mathrm{n}^{\circ} 72508 / 13$, $\$ 106$ 
[...] dissuader d'autres partisans d'opposition et le grand public de participer à des manifestations et, plus généralement, à un débat politique ouvert. L'effet dissuasif des sanctions a été accru par le fait que celles-ci visaient un personnage public bien connu, dont la privation de liberté devait nécessairement attirer l'attention des médias ${ }^{98}$.

De même, une détention préventive ne peut être justifiée sans preuves par la simple appartenance à une association d'aide aux étudiants, soupçonnée de liens avec une organisation criminelle, dont les membres présumés sont accusés d'avoir mené des activités visant le renversement du gouvernement par la force et la violence, et de préparer un coup d'État militaire ${ }^{99}$. D'une manière plus générale encore, la Cour a été amenée à rappeler le caractère exceptionnel du recours à la prison à l'égard des mineurs, qui ne peut être qu'une mesure de dernier ressort et d'une durée aussi brève que possible ${ }^{100}$.
Libérale et volontairement orientée vers la protection des personnes privées de liberté, la jurisprudence de la Cour européenne des droits de l'homme l'est sans aucun doute. Mais elle participe surtout d'une perspective très large qui vise à éviter la systématisation du recours à la prison, car il convient toujours pour les autorités nationales d'envisager l'application de mesures moins sévères que la privation de liberté. Il ne faut par ailleurs jamais oublier que, même privée de sa liberté, la personne incarcérée n'en demeure pas moins membre de la communauté, puisque la Cour rappelle inlassablement que c'est aujourd'hui sur l'objectif de réinsertion

[...] que les politiques pénales européennes mettent désormais l'accent, ainsi qu'il ressort de la pratique des États contractants, des normes pertinentes adoptées par le Conseil de l'Europe et des instruments internationaux applicables ${ }^{101}$.

98. Cour EDH, 13 décembre 2016, Kasparov et autres c. Russie ( $\left.n^{\circ} 2\right), \mathrm{n}^{\circ}$ 51988/o7, $\$ 32$.

99. Cour EDH, 31 mai 2016, Mergen et autres c. Turquie, $\mathrm{n}^{\circ}$ 44062/o9, 55832/o9, 55834/o9, 55841/o9, 55844/o9 et Ayşe Yüksel et autres c. Turquie, $\mathrm{n}^{\mathrm{o}} 55835 / 09,55836 / 09,55839 / 09$. En l'occurrence l'Association turque de soutien à la vie moderne (Çağdaş Yaşamı Destekleme Derneǧi - ÇYDD), structure attribuant des bourses aux étudiants (notamment pour promouvoir l'éducation des jeunes filles) et soupçonnée d'être liée à une organisation criminelle dénommée Ergenekon.

100. Cour EDH, 19 janvier 2016, Gülcü c. Turquie, n 17526/10.

101. Cour EDH, GC, 17 janvier 2017, Hutchinson c. Royaume-Uni, $\$ 42$. 